\title{
Targeting angiogenesis in myocardial infarction: Novel therapeutics (Review)
}

\author{
JIEJIE LI, YUANYUAN ZHAO and WEI ZHU \\ Jiangsu Key Laboratory of Medical Science and Laboratory of Medicine, \\ School of Medicine, Jiangsu University, Zhenjiang, Jiangsu 212013, P.R. China
}

Received August 25, 2021; Accepted November 1, 2021

DOI: $10.3892 /$ etm.2021.10986

\begin{abstract}
Acute myocardial infarction (AMI) remains the main cause of mortality worldwide. Despite surgery and medical treatment, the non-regeneration of dead cardiomyocytes and the limited contractile ability of scar tissue can lead to heart failure. Therefore, restoring blood flow in the infarcted area is important for the repair of myocardial injury. The objective of the present review was to summarize the factors influencing angiogenesis after AMI, and to describe the application of angiogenesis for cardiac repair. Collectively, this review may be helpful for relevant studies and to provide insight into future therapeutic applications in clinical practice.
\end{abstract}

\section{Contents}

1. Introduction

2. Regulation of angiogenesis after AMI

3. Application of angiogenesis for cardiac repair

4. Discussion

\section{Introduction}

Acute myocardial infarction (AMI) is myocardial necrosis caused by acute and persistent ischemia and hypoxia in the coronary arteries (1). There is an urgent need for improved treatment strategies. The traditional treatment for AMI is mainly surgery or drug therapy, including thrombolytic therapy, percutaneous coronary intervention (PCI) and coronary artery bypass graft (CABG) (2). These traditional treatment methods can save dying cardiomyocytes, decrease the area of infarction progression and delay myocardial remodeling.

Correspondence to: Ms. Yuanyuan Zhao, Jiangsu Key Laboratory of Medical Science and Laboratory of Medicine, School of Medicine, Jiangsu University, 301 Xuefu Road, Zhenjiang, Jiangsu 212013, P.R. China

E-mail: 1000004441@ujs.edu.cn

Key words: angiogenesis, acute myocardial infarction, vascular endothelial cells, exosomes, mesenchymal stem cells
However, they do not promote myocardial cell regeneration, and the contractile ability of the scar tissue in the infarcted is limited; therefore, the myocardial contractile force is gradually reduced, causing myocardial fibrosis, arrhythmia and ventricular diastolic dysfunction, leading to advanced congestive heart failure (3). Due to the limits of traditional methods, significant research has been devoted to post-infarction repair. Angiogenesis is essential for correct healing post-infarction. The blood supply of the cells in the infarcted area gradually decreases, which restricts oxygen transfer, nutrient absorption and removal of metabolic waste, and the cardiomyocytes gradually become necrotic; therefore, restoring the blood supply to the infarcted area is a favorable repair method (4). The present review outlines the progress of current research on angiogenesis in myocardial infarction repair, including the main factors affecting angiogenesis and the therapeutic methods.

\section{Regulation of angiogenesis after AMI}

Angiogenesis is the formation of new blood vessels based on previous vasculature. The formation of blood vessels starts with the sprouting of endothelial cells (ECs), which adhere to each other and are connected to the extracellular matrix (ECM), followed by hydrolytic remodeling of ECM in the presence of various enzymes. Hydrolytic remodeling of ECM refers to the continuous process of decomposition and synthesis of the ECM under the action of various enzymes (5). There are three main types of ECs, namely tip, stalk and phalanx cells (6). Tip and stalk cells are located at the sprouting tip of blood vessels and can secrete a variety of pro-angiogenic factors, such as vascular endothelial growth factor (VEGF), fibroblast growth factor and platelet-derived growth factor (PDGF) (7). There are numerous factors that affect the formation of blood vessels.

Factors driving angiogenesis after AMI. After AMI, the repair process of cardiac injury begins with the inflammatory phase. This is characterized by the activation of natural immune pathways and the recruitment of inflammatory leukocytes to remove dead cells from the wound, which involves the complement system, reactive oxygen species and the participation of various chemokines (8). Some other cells are also activated and involved in repair, such as macrophages, fibroblasts, ECs, lymphocytes and other immune cells (9). 
Macrophages transfer to a pro-angiogenic phenotype after Annexin A1 treatment (10). Moreover, hyaluronic acid (HA) is a major component of the ECM, and is a non-protein extracellular molecule. HA oligosaccharides (o-HA; $<10$ disaccharides units) is a partially degraded fragment of high molecular weight HA. Cardiac o-HA improves polarization of M2 macrophages, and prevents the inflammatory response caused by neutrophils and accelerates restitution of myocardial function (11).

Fibroblasts are the main cellular component of myocardial injury. They are derived from embryonic mesenchymal cells and can produce collagen and other proteins. Fibroblasts have multiple phenotypes, and some populations express fibroblast-specific protein (FSP) 1 and $\alpha$-smooth muscle actin ( $\alpha$-SMA). FSP1-positive fibroblasts contribute to angiogenesis and repair, and their reparative effect is greater than that of $\alpha$-SMA-positive fibroblasts (12). Moreover, as infarction progresses, fibroblast function transforms from inflammation to angiogenesis (13).

Vascular ECs are simple squamous epithelial cells that line the inner surface of blood vessels. Hypoxia is a potent angiogenic stimulus during cardiac repair (Fig. 1). Hypoxia transcriptionally upregulates angiogenic integrins in microvascular ECs and promotes the migration and tube formation of HMEC-1 cells (14). Furthermore, hypoxia can induce a large number of vascular-related signaling pathways in ECs to upregulate and promote angiogenesis, such as hypoxia-inducible factor (HIF)-1 (15). HIF-1 $\alpha$ binds to the promoter of Twist1 to activate Twist1 transcription and regulate endothelial-mesenchymal transition (16). It has been reported that neutrophils and mast cells promote angiogenesis $(17,18)$. Furthermore, neutrophil extracellular traps produced by dead neutrophils promote inflammatory angiogenesis in vivo and in vitro (19). It has also been shown that mast cells can release some pro-angiogenic cytokines, such as PDGF and VEGF (20).

There are several molecules involved in angiogenesis. Under the conditions of ischemia and hypoxia, numerous cells secrete additional pro-angiogenic factors. For instance, VEGF plays an important role in angiogenesis. VEGF is upregulated by HIF- $1 \alpha$ and regulates angiogenesis by binding to a specific receptor, VEGFR, and soluble VEGFR1 (sVEGFR1) is secreted out of the cell to participate in the sprouting process of new blood vessels (21). HIF-1 $\alpha$ and VEGF are closely associated with Notch signaling (22). Notch and Notch ligand $\delta$-like (Dll)4 signaling is related to angiogenesis (23). VEGFA activates the membrane-bound ligand Dll4 of tip cells and transmits D114 signals to nearby ECs (24). The angiogenin family and hepatocyte growth factor (HGF) also contribute to angiogenesis. $\mathrm{HGF} / \mathrm{Met}$ induces the proliferation and migration of ECs via Ras-related C3 botulinum toxin substrate 1 activation. In fibroblasts, HGF/Met antagonizes the actions of TGF- $\beta 1$ and angiotensin II, thereby preventing fibrosis. HGF/Met also influences the inflammatory response of macrophages and the immune response of dendritic cells, indicating their protective function against atherosclerotic and autoimmune diseases (25). In addition, recombinant protein has been widely used as a molecule to promote angiogenesis. For instance, the recombinant human PDGF antibody promotes the repair of cardiac wounds after myocardial infarction by changing the mechanical mechanism of infarction scarring, thus improving cardiac function, reducing ventricular arrhythmia and improving survival rate (26).
Factors inhibiting angiogenesis after AMI. There are fewer anti-angiogenic than pro-angiogenic factors, including cells, secreted factors and recombinant proteins. M1-like macrophage-derived exosomes suppress angiogenesis in a myocardial infarction microenvironment, which may be related to microRNA (miRNA/miR)-155 in exosomes (27). Moreover, 11 $\beta$-hydroxysteroid-1 in macrophages can inhibit inflammatory angiogenesis (28). VEGF-A165b is an anti-angiogenic factor that as has been identified as a regulator of vascularization (29). In addition, the anti-angiogenic pigment epithelium-derived factor suppresses angiogenesis in the human heart by inhibiting VEGF-induced sprouting (30). Similarly, Ly6/Plaur domain-containing 1 is a novel antiangiogenic factor derived from human cardiac fibroblasts, which suppresses EC network formation (31). Furthermore, Wnt/ $\beta$-catenin signaling plays an important role in angiogenesis. The transcription factor BTB and $\mathrm{CNC}$ homology 1 impairs angiogenesis after ischemic injury by suppressing Wnt/ $\beta$-catenin signaling (32). It has also been shown that recombinant human IL-24 can suppress tumor angiogenesis $(33,34)$. However, to the best of our knowledge, IL-24 has not been studied in the repair of myocardial injury. IL-12 is also an anti-angiogenic factor that is mainly produced by $\mathrm{CD} 11 \mathrm{~b}(+)$ monocytes in mice after MI. In addition, IL-12 affects the formation of blood vessels in the yolk sac, which can retard embryonic development (35).

\section{Application of angiogenesis for cardiac repair}

Mesenchymal stem cells (MSCs). MSCs are stromal cells that have self-renewal ability and show multilineage differentiation. MSCs can be isolated from a variety of tissues, such as umbilical cord, endometrial polyps, menses blood, stem cells, bone marrow and adipose tissue $(36,37)$. Due to their powerful function and easy access, MSCs have been widely used by researchers in recent years, especially in the study of ischemic heart disease (38). For example, placenta-derived MSCs can be used to promote therapeutic angiogenesis. Such MSCs can differentiate into vascular-like cells and secrete some provascular factors to promote angiogenesis. These cytokines include VEGF, basic fibroblast growth factor, IL-6, IL-8, HGF, insulin-like growth factor (IGF) binding protein (IGFBP)2, IGFBP3 and IGFBP6. These factors generate blood vessels by activating key provascular-related signaling pathways (39).

MSCs from fat and bone marrow promote angiogenesis via unique cytokines and protease expression mechanisms. Adipose-derived stem cells promote utilization of the plasminogen activator/plasmin axis by ECs as the primary means of vessel invasion and elongation in fibrin (40). MMPs serve a purpose in regulating capillary diameter and possibly in stabilizing the nascent vessels (40). MMPs also play an important role in the differentiation of stromal stem cells (41). MSCs can regulate expression of MMP9 (42). Therefore, MSCs and MMPs may have important roles in angiogenesis after AMI. In addition to the role of MSCs, it has been proposed that the functional benefits observed after MSC transplantation in experimental models of tissue injury may be associated with the secretion of soluble factors acting in a paracrine fashion $(43,44)$. Moreover, stem cells also play an important role in the repair of other tissues. MSCs derived from the umbilical cord can relieve limb ischemia via the formation of blood vessels in mice (45). 


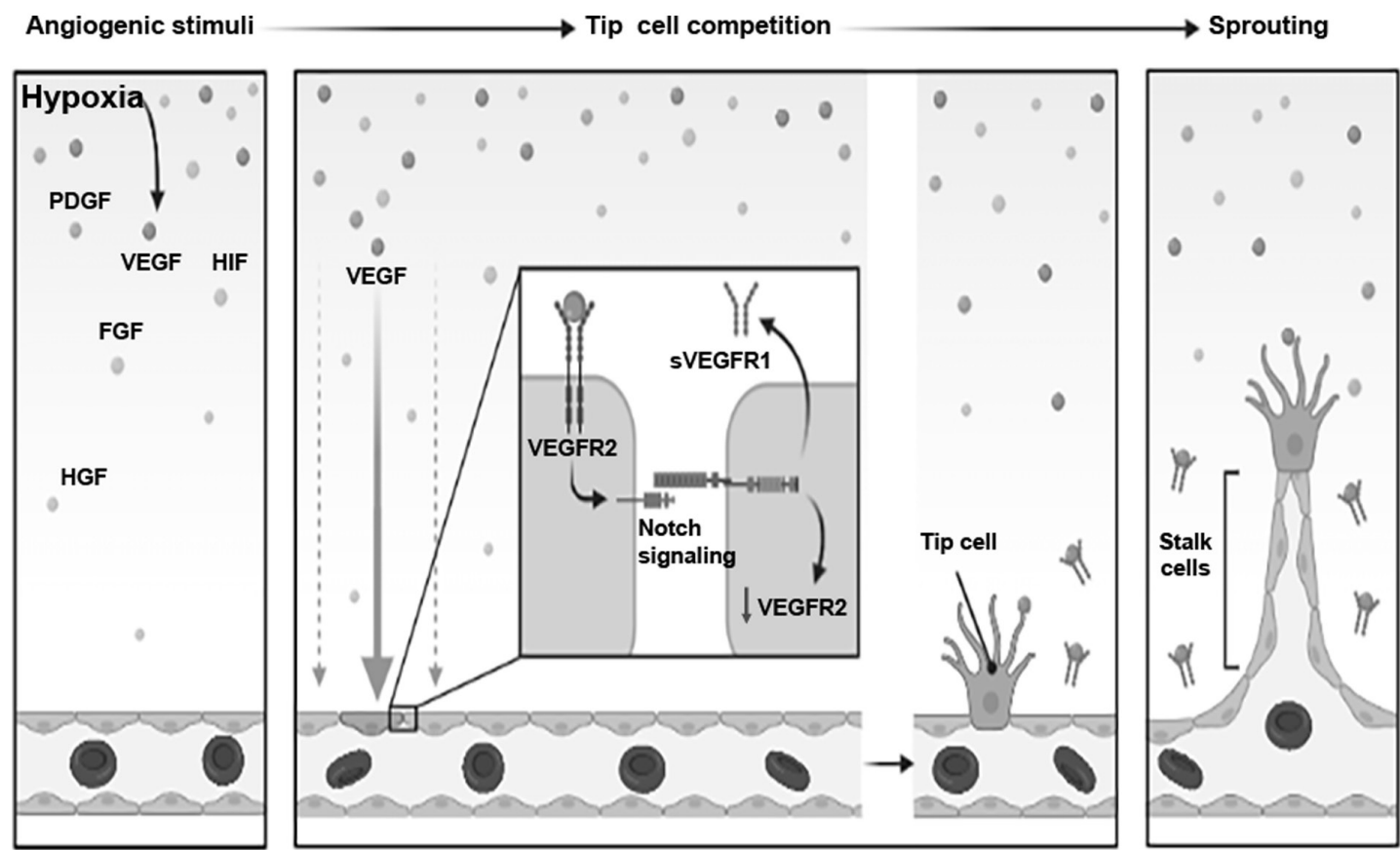

Figure 1. Hypoxia stimulation can produce a variety of pro-angiogenic factors. Among them, VEGF can bind to VEGFR2 of endothelial cells via the Notch signaling pathway to downregulate VEGFR2 and secrete sVEGFR1. Moreover, endothelial cells preferentially transform into Tip cells and combine with Stalk cells to form endothelial cells sprouting process. sVEGFR1, soluble VEGFR1; HIF, hypoxia-inducible factor; FGF, fibroblast growth factor receptors; HGF, hepatocyte growth factor.

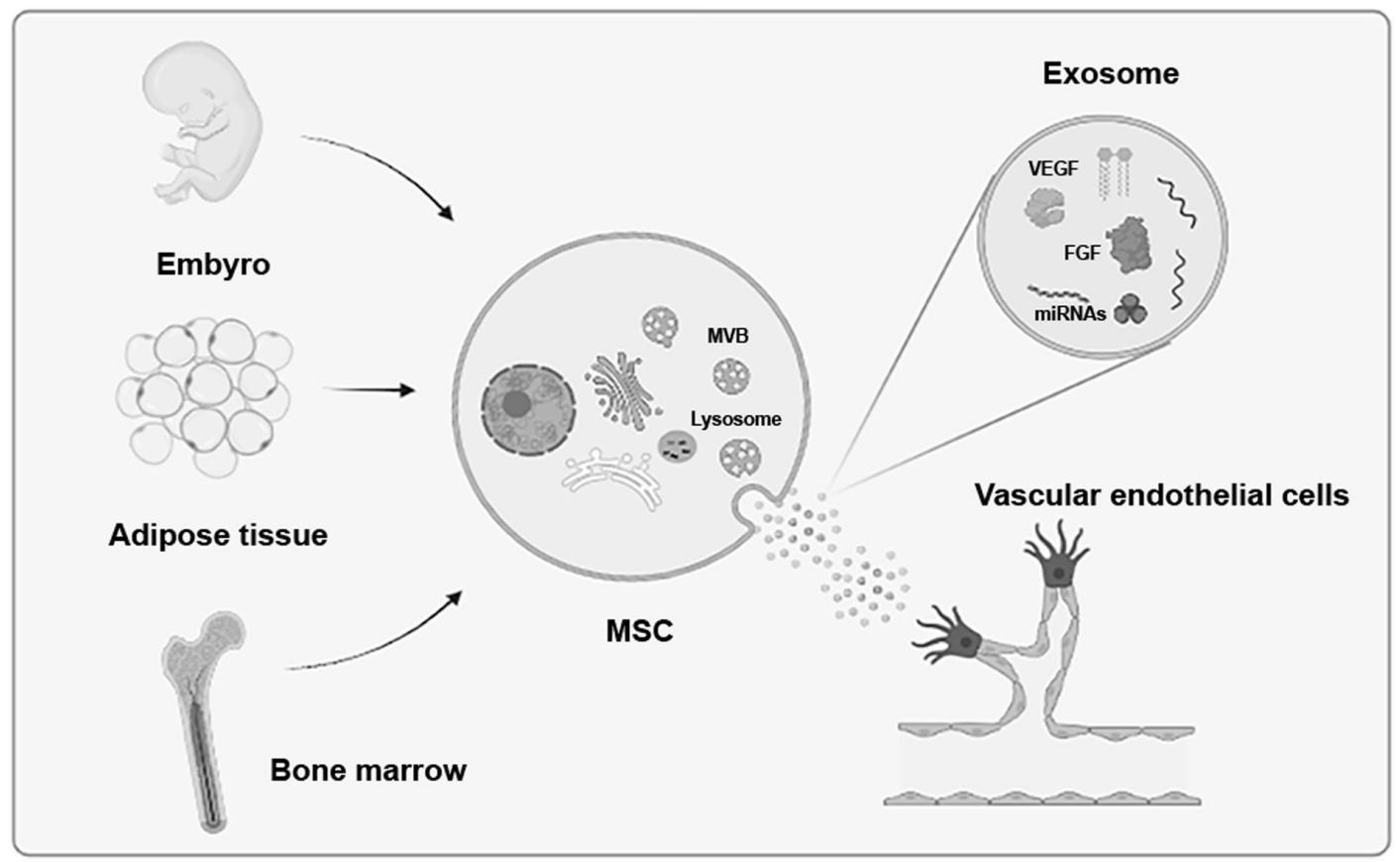

Figure 2. Main sources and packaging release process of exosomes. Exosomes are mainly derived from MSCs isolated from embryos, adipose tissue and bone marrow. The cell membrane invades to form MVB. Some of the multivesicular bodies are degraded by lysosomes through the Golgi apparatus, and some are fused with the plasma membrane and released outside the cell. Exosomes contain several angiogenic molecules, including miRNAs and proteins, such as VEGF and FGF. MSC, mesenchymal stem cell; miRNA, microRNA; FGF, fibroblast growth factor receptors; MVB, multivesicular bodies.

It has also been shown that human neural stem cells promote the proliferation of endogenous neural stem cells and enhance angiogenesis in the brain of ischemic rats (46).

Exosomes. Exosomes are small extracellular vesicles that are only $50-150 \mathrm{~nm}$ in diameter, surrounded by a lipid bilayer membrane and contain components derived from their original cells (47). Exosomes have a relatively rich source, existing in various tissues and cells throughout the body, such as embryos, adipose tissue and bone marrow (Fig. 2).

The extraction methods for exosomes include ultracentrifugation, immunoprecipitation, size-based isolation 
techniques and commercial rapid extraction reagents. The most widely used methods are ultracentrifugation and rapid extraction reagents (48). These methods have both advantages and disadvantages, and thus the appropriate method to extract exosomes should be selected according to the research needs. Another important point is the identification of exosomes, and the quality of exosomes plays a crucial role in research (49). As early as 2014, the International Association of Extracellular Vesicles proposed that the identification of exosomes can be divided into three levels: Transmission electron microscopy (TEM), nanosight particle size and protein markers (50). In general identification, there must be $\geq 3$ vesicle-positive protein makers, including $\geq 1$ transmembrane or lipid-binding protein and one cytoplasmic protein, $\geq 1$ vesicle-negative protein maker. The identification of a single vesicle requires two different but complementary methods, such as TEM or atomic force microscopy plus nanoparticle tracking analysis (50).

Endothelial progenitor cells (EPCs) were first discovered in peripheral blood (51). Some studies have found that EPCs also participate in the process of angiogenesis by releasing exosomes (52-54). EPC-derived exosomes promote the proliferation and angiogenesis of cardiac fibroblasts by inhibiting mesenchymal-endothelial transition and decreasing the expression of high mobility group protein 1 (55). Furthermore, EPC-derived exosomes are newly found players in the beneficial effects of exercise on vascular diseases. For instance, moderate exercise enhances the function of circulating EPC-derived exosomes in protecting ECs against hypoxic injury, and which may enrich EPC-derived exosomes via the release of miR-126 (56).

Circular RNA (circRNA/circ) in exosomes also plays an important role in tissue repair. Cardiomyocytes subjected to hypoxia release circ_homeodomain-interacting protein kinase 3 (circHIPK3)-rich exosomes to regulate oxidative stress damage in cardiac ECs, and circHIPK3 increases the expression of VEGFA by inhibiting the activity of miR-29a, thereby promoting the acceleration and proliferation of cardiac ECs (57). It has been confirmed that exosomal circHIPK3 released from hypoxia-pretreated cardiomyocytes regulates oxidative damage in cardiac microvascular ECs via the miR-29a/IGF-1 pathway (58). circHIPK3 downregulates miR-421, resulting in increased Forkhead box (FOX)O3a expression, thereby inhibiting apoptosis and inhibiting release of IL-1 antioxidant protein and IL-18, ultimately repairing ischemic injury (59).

Previous research has shown that exosomes from patients with myocardial ischemia promote angiogenesis via the miR-939/inducible nitric oxide (NO) synthase/NO pathway (60). There have been similar studies in skin repair (61-63). Moreover, it has been shown that exosomes from the serum of patients with type 2 diabetes can hinder the process of injury repair, and this effect is related to the miR-20b-5p and $\mathrm{Wnt} 9 \mathrm{~b} / \beta$-catenin pathways (64).

Some researchers have taken on a new approach and focused on exosomes themselves. This approach can achieve targeted repair of injury sites through exosome modification. It was found that tissue inhibitor of metalloproteinase-2-modified human umbilical cord MSC-derived exosomes enhanced the repair of myocardial infarction in a rat model via the Akt/secreted frizzled related protein 2 pathway (65). Moreover, overexpression of HIF-1 $\alpha$ in MSC-derived exosomes can enhance angiogenesis after AMI (66). Stromal cell derived factor (SDF)-1 overexpression in MSC-derived exosomes inhibited autophagy of ischemic myocardial cells and promoted microvascular production of ECs (67).

At present, the application of engineered exosomes in injury repair is still the mainstream direction. Exosomes engineered by ischemic myocardium-targeting peptide (IMTP) CSTSMLKAC can specifically target ischemic myocardium, and MSC-derived-IMTP-exosomes exert enhanced therapeutic effects on AMI (68). CSTSMLKAC is a new peptide sequence that can preferentially target the ischemic area of the heart (68). miRNA can also be used to modify exosomes. miR-322-modified, cardiac-progenitor-cell-derived exosomes provide protection against MI via Nox2-dependent angiogenesis (69). Moreover, exosomes derived from miR-146a-modified adipose-derived stem cells can downregulate early growth response factor 1 to attenuate AMI-induced myocardial damage (70).

In addition, with controlled-release properties, drug-carrying nanoparticle (NP) systems are expected to enhance cardiac protection in patients with cardiac ischemic events. NPs can provide sustained and precise exposure of the infarcted area through direct intramuscular or intravenous injection with drugs with active targets (71). There are also exosomes from MSCs modified with mononuclear cell mimics, which have a high targeting efficiency for injured myocardium and promote endothelial maturation during angiogenesis (72). In cardiac repair, NPs mainly play a role by modifying exosomes and acting as nano drug delivery systems (73).

Some novel ideas and methods have emerged from research into repair of other organs. By constructing a CD9-HuR protein, a new exosome was designed, which has a strong ability to enrich specific RNAs, effectively delivering RNA into cells, targeting genes in vivo and in vitro, and treating liver disease in a mouse model (74). Researchers from Switzerland have reported a series of synthetic biology-inspired control devices that are known as EXOsomal Transfer Into Cells devices, which serve to enhance these steps, enabling efficient exosomal mRNA delivery without the need to concentrate exosomes. This design of exosomes reduces the neurotoxicity and neuroinflammation of Parkinson's disease via the delivery of therapeutic catalase mRNA (75).

Biomaterials. In recent years, additional research has focused on repair by using biomaterials (76). Compared with traditional cell therapy, biomaterials have more advantages, such as being degradable, easy to obtain and free to regulate the repair process. Biomaterials are mainly divided into natural and artificial synthetic materials. Natural materials mainly refer to the various components of mammalian ECM, such as collagen, fibrinogen, Matrigel and gelatin. Natural materials also include some ingredients extracted from plants or animals, such as chitosan and cellulose. Artificial synthetic materials are easier to obtain and are more plastic (77).

Biomaterials can be combined with cells or factors to promote wound healing $(78,79)$ (Fig. 3). Biomaterials with loaded stem cells and immunomodulating and tissue-regenerating factors can be used to ameliorate inflammation, improve 


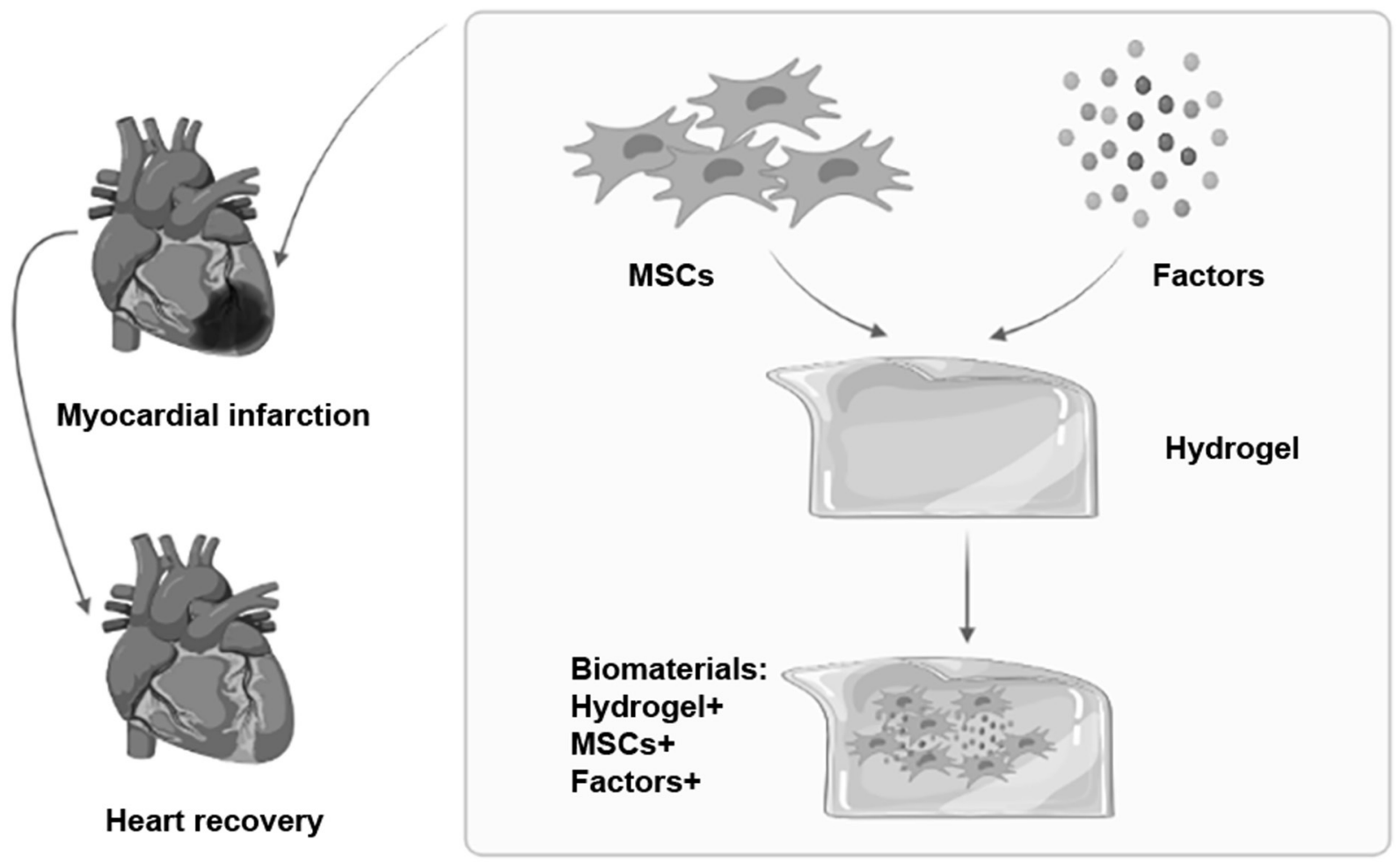

Figure 3. Application of biomaterials for cardiac repair. MSCs or pro-angiogenic factors can be combined with hydrogels to form biomaterials with improved repair performance, and can be targeted to the site of heart injury by injection, implantation and other ways to form new blood vessels. MSC, mesenchymal stem cell.

angiogenesis, reduce fibrosis and generate functional cardiac tissue (78). For example, the encapsulation of VEGF in polylactic acid glycolic acid NPs improves the therapeutic efficacy of VEGF and promotes angiogenesis (79). Liu et al (80) reported that co-transplantation of chitosan thermosensitive hydrogel and bone-marrow-derived MSCs can ameliorate the inflammatory response and promote cardiac functional recovery. Citrate hydrogels can be injected as an angiogenic biomaterial to improve cardiac function after AMI and increase the number of blood vessels in the infarcted area (81). In addition to the injectable biomaterials, some researchers have developed thermal plastic poly (glycolic acid) surgical sutures and mussel-inspired conductive particle adhesion into highly elastic, conductive spring-like coils. The polypyrrole-coated biospring acts as an electrode and is assembled into a solid-state supercapacitor. After being injected through a syringe needle $(0.33-\mathrm{mm}$ inner diameter), the tangled coils form an elastically conductive three-dimensional (3D) network to modulate angiogenesis (82). Additionally, a cardiopatch has been created with adipose-tissue-derived progenitor cells seeded into an engineered bioimplant consisting of 3D bioabsorbable polycaprolactone scaffolds filled with a peptide hydrogel. This treatment decreases fibrosis, limits infarct scar expansion and reduces postischemic ventricular deformity (83). Similarly, the use of chitosan/calcium silicate heart patches showed increased angiogenesis in rats after myocardial infarction (84). The alginate scaffold modified with RGDfK (Arg-Gly-Asp-D-Phe-Lys) peptide can be used for cell transplantation and cardiac neovascularization (85). It is also interesting to note that by changing the stiffness of hydrogel, VEGF secretion of MSCs can be improved, thus further promoting vascular formation (86).
In addition to using biomaterials to generate new blood vessels, artificial blood vessels have already been constructed to treat injuries. The raw materials for manufacturing artificial blood vessels are polyester, polytetrafluoroethylene, polyurethane and natural mulberry silk. Artificial blood vessels supported by spider silk can be constructed in vitro (87). Moreover, small-diameter artificial blood vessels can promote in situ endothelialization (88). Remarkably, artificial blood vessels are rarely used in cardiac injury. Therefore, small-diameter artificial blood vessels are expected to be applied in cardiac repair in the future.

Biological factors. Some biological factors play a role in the repair of AMI, and an increasing number been applied in recent years (89). Biological factors are partly obtained from artificial synthesis. SDF-1 is a distinctive cytokine that can protect the heart from ischemic injury. Annexin V can accurately detect dead cells in the body. SDF-1 and Annexin V in combination can reduce apoptosis, increase angiogenesis, reduce infarcted area and improve heart function in mice after AMI (90).

Some small-molecule hormones also play an important role in injury repair. For instance, $\beta$-estradiol promotes recovery after AMI by enhancing the homing and angiogenesis of bone-marrow-derived EPCs by enhancing estrogen receptor/SDF-1/C-X-C motif chemokine receptor 4 crosstalk (91). Hormones also promote myocardial repair after infarction. Dihydrotestosterone induces angiogenic factors and helps to nest MSCs into heart tissue (92). Furthermore, irisin plays an anti-MI role by promoting angiogenesis (93).

A number of other small molecules have restorative and therapeutic effects. New collagenous stromal elements reduce left ventricular dilatation after MI by promoting scar formation and angiogenesis (94). Enhanced extracellular sulfatase with 
Table I. Application of angiogenesis for cardiac repair.

\begin{tabular}{|c|c|c|c|}
\hline Therapy & Source & Mechanism & (Ref.) \\
\hline MSCs & $\begin{array}{l}\text { Umbilical cord, bone } \\
\text { marrow, adipose tissue, } \\
\text { menses blood, } \\
\text { other tissues }\end{array}$ & $\begin{array}{l}\text { Secret pro-angiogenic factors } \\
\text { Regulate endothelial cells } \\
\text { Regulate MMP expression } \\
\text { Regulate cardiac fibroblasts }\end{array}$ & $\begin{array}{l}(39) \\
(40) \\
(42) \\
(55)\end{array}$ \\
\hline Exosomes & $\begin{array}{l}\text { Embryos, ADSC } \\
\text { BM-MSC, EPC, Serum } \\
\text { Engineered exosomes } \\
\text { Other tissues }\end{array}$ & $\begin{array}{l}\text { Protect ECs against hypoxia injury } \\
\text { Deliver and regulate circRNAs/miRNAs } \\
\text { to target cells } \\
\text { Involve in miR-939/iNOS/NO pathway } \\
\text { Protein modification, overexpressed } \\
\text { protein and miRNA }\end{array}$ & $\begin{array}{c}(56) \\
(57) \\
(60) \\
(65-72,74)\end{array}$ \\
\hline Biomaterials & $\begin{array}{l}\text { Natural materials: } \\
\text { Collagen, fibrinogen, } \\
\text { Matrigel, gelatin, etc. } \\
\text { Artificial synthetic }\end{array}$ & $\begin{array}{l}\text { Ameliorate inflammation, } \\
\text { reduce fibrosis, and generate } \\
\text { functional cardiac tissue } \\
\text { Promote angiogenesis }\end{array}$ & $\begin{array}{r}(78,80,83) \\
(81,82,84-86)\end{array}$ \\
\hline Biological factors & $\begin{array}{l}\text { Artificial synthesis } \\
\text { Animal or plant } \\
\text { extraction }\end{array}$ & $\begin{array}{l}\text { Reduce apoptosis, increase angiogenesis, } \\
\text { reduce infarcted area } \\
\text { Induce angiogenic factors }\end{array}$ & $(90-98)$ \\
\hline Gene therapy & $\begin{array}{l}\text { Genetic engineering } \\
\text { technology }\end{array}$ & $\begin{array}{l}\text { Construct angiogenic fusion plasmid } \\
\text { Angiogenic gene modified cells }\end{array}$ & $\begin{array}{c}(101) \\
(103,105,107)\end{array}$ \\
\hline
\end{tabular}

MSCs, mesenchymal stem cells; ADSC, adipose-derived MSC; BM-MSC, bone marrow MSC; ECs, endothelial cells; iNOS, inducible nitric oxide synthase; NO, nitric oxide; circRNA, circular RNA; miRNA/miR, microRNA.

heparan sulfate enhances the bioavailability of VEGF during ischemic heart repair, thereby promoting angiogenesis (95). circRNAs are also involved in myocardial repair. After AMI, adeno-associated-virus-9-mediated circ_fibronectin type III domain containing $3 \mathrm{~B}$ overexpression in the heart can reduce myocardial apoptosis, enhance the formation of new blood vessels and improve left ventricular function (96).

Some Chinese herbal extracts also play an important role in injury repair. For example, Catalpa extracted from traditional Chinese medicine has been proven to promote angiogenesis and VEGF expression in ischemic myocardium (97). Chinese medicine GeGen-DanShen extract protects against myocardial ischemic injury by promoting angiogenesis via the upregulation of the VEGF/VEGFR2 signaling pathway (98). Moreover, the Ginkgo biloba extract can suppress the inflammation- and apoptosis-regulating p38 MAPKs, NF- $\kappa \mathrm{B}$ and $\mathrm{Bcl}-2$ signaling pathways to prevent AMI (Fig. 4) (99).

Gene therapy. Gene therapy is an emerging technology. Elabela (ELA) is a newly discovered hormone peptide containing 32 amino acids, which is known to regulate endodermal differentiation and cardiovascular development (100). Jin et al (101) successfully constructed a p-adeno-associated virus-3 x Flag/ELA-32 fusion expression plasmid using molecular cloning technology. Their results showed that this fusion plasmid can promote angiogenesis after MI (101). Gene and stem cell therapies also hold promise for the treatment of ischemic cardiovascular disease. The combination of human cord blood CD34(+) cells and Ang1 and VEGF genes promotes angiogenesis and reduces infarct size (102). Genetic engineering technology is also widely used to modify cells. FOXO transcription factors can modulate endothelial gene expression and function (103). Human vascular ECs can be functionally enhanced by engineering them to express an activated form of FOXO3 (104). Heme oxygenase (HO)-1 is a cytoprotective, pro-angiogenic and anti-inflammatory enzyme. Human placental MSCs modified with HO-1 can promote placental angiogenesis by improving the balance of angiogenic factors (105). In ischemic skeletal muscle, human adipose-derived stromal cells expressing VEGF165 can promote angiogenesis (106). Clustered regularly interspaced short palindromic repeats (CRISPR)/Cas9 has become a powerful technology to modify cells. Several studies have used CRISPR/Cas9 to edit cells to promote injury of tissues or organs. Deletion of enhancers and long non-coding RNAs by CRISPR/Cas9 promotes significant changes in VEGFA and VEGFC expression in ECs (107). Moreover, CRISPR/Cas9 gene therapy based on TGF- $\beta 1$ alleviates radiation-induced lung injury (108). Although CRISPR/Cas9 is rarely used in cardiac repair, it can be expected in the future. The application of MSCs, exosomes, biomaterials, biological factors and gene therapy in angiogenesis for cardiac repair was summarized in Table I.

\section{Discussion}

Promoting angiogenesis in the infarcted area is the key to the treatment of sequelae of AMI. Restoring the blood supply 


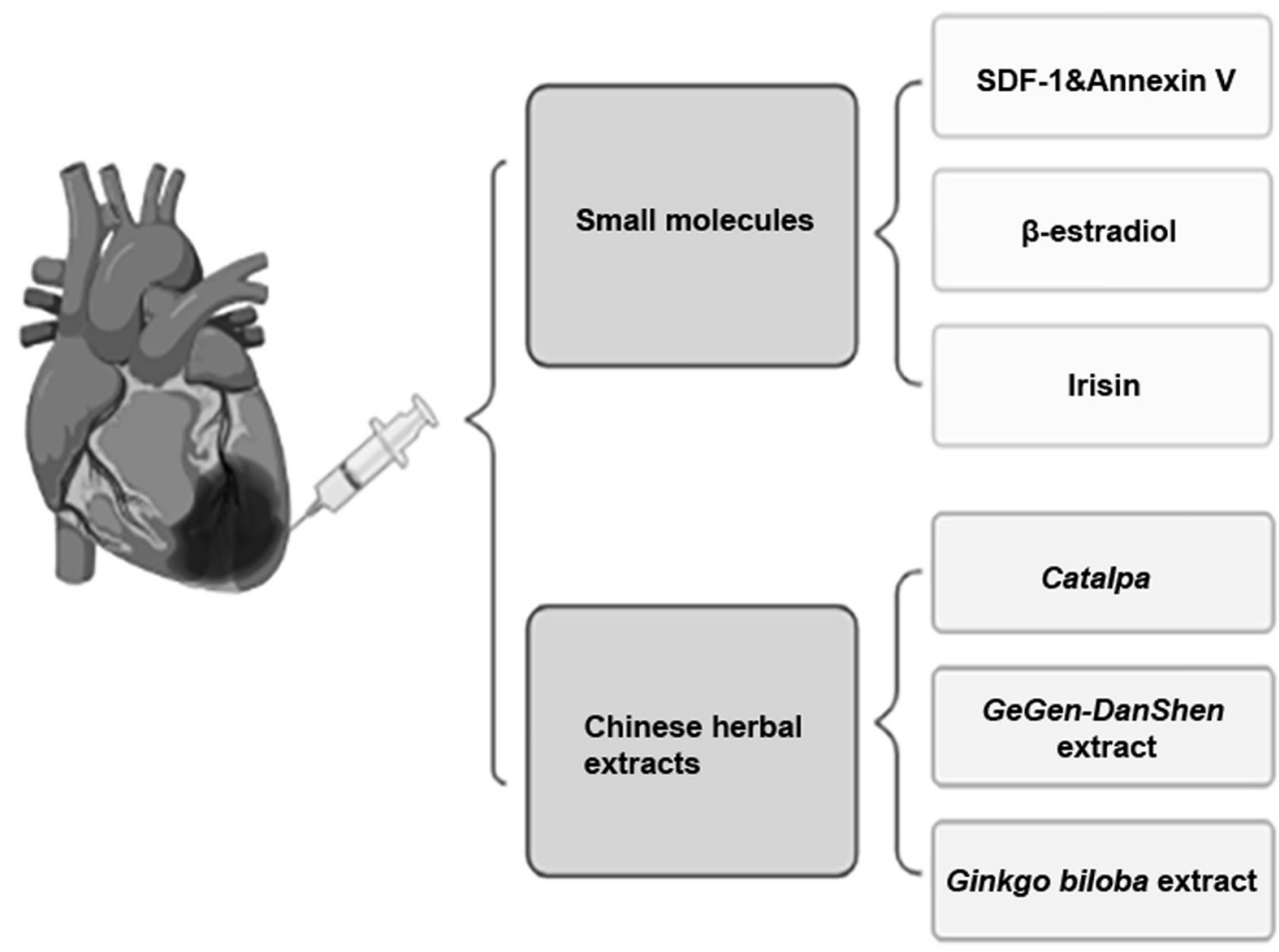

Heparan sulfate

\section{Dihydrotestosterone}

\section{Collagenous stromal} elements

Figure 4. Biological factors for the treatment of myocardial infarction. The figure mainly shows two types of biological factors for cardiac repair. The small molecules listed are SDF-1, Annexin V, $\beta$-estradiol, irisin, heparan sulfate, dihydrotestosterone and collagenous stromal elements. Catalpa, GeGen-DanShen extract and Ginkgo biloba extract are all Chinese herbal extracts. SDF-1, stromal cell derived factor.

to this area can effectively reduce the area of cardiac tissue necrosis, which in turn can improve cardiac function and quality of life (109). Although traditional treatments such as PCI and CABG have numerous advantages, there are also several complications that are difficult to overcome (110). The common complications of CABG include postoperative bleeding and acute coronary artery occlusion, which can lead to severe or even critical conditions (111).

By contrast, the new therapeutic methods mentioned in this review have greater repair potential. MSCs have superior therapeutic effects, low antigenicity, wide application and rich sources. Furthermore, the safety of MSCs has been demonstrated in clinical trials (112). Nevertheless, some of the problems with treatment with MSCs also need to be resolved. For example, MSCs are easily differentiated, heterogeneous and diverse in large-scale production. Furthermore, MSCs easily die when transplanted into the body and, in cases, cannot adapt to the microenvironment (113). In response to these problems, further research and development of new technologies and methods are required to achieve precise and efficient repairs.

Exosomes have the advantages of being more stable, capable of mass production, controllable and easy to store. Unlike MSCs, exosomes can maintain biological activity for a long time in vitro, thereby overcoming the short-term apoptotic characteristics of MSCs. In addition, exosomes carry more diversified information, which can be used for early diagnosis, relapse monitoring, and drug resistance monitoring (114). Even so, numerous problems still need to be resolved. For instance, the separation and purification technology of exosomes is not yet mature, and there is no unified standard for separation and analysis, to the best of our knowledge. Otherwise, the identification of exosomes as disease markers still depends on a large number of clinical trials (115).

Biomaterials have been applied clinically for a long time. He et al (116) were the first to achieve a major breakthrough in the clinical study of injectable scaffold materials combined with stem cell transplantation for the treatment of ischemic heart disease, and proved its clinical safety and feasibility. Although biomaterials have benefits, there are still several problems, such as toxicity, cost and effectiveness. The application prospects for biological factors should not be underestimated. Several biological agents have already been applied clinically but there are also some drawbacks, such as adverse effects, easy inactivation and cost (117).

Biological factors and gene therapy also play an important role in cardiac repair. Biological factors are more effective, but at the same time, they have the problems of short half-life and easy inactivation. Therefore, new technology is required to resolve these problems. After $>10$ years of development, research on gene therapy has made significant progress. However, it is still in the early stage of clinical trials, and cannot guarantee stable efficacy and safety. Despite the numerous obstacles, the trend towards gene therapy is encouraging.

In view of all previous studies on angiogenesis in AMI, it remains necessary to explore the therapeutic mechanisms mediated by these novel methods, especially when the molecules that play an effective role in the disease have not yet been identified. Although MSCs and exosomes face several challenges in industrialization, their functional research, diagnosis and treatment applications in some diseases, such as AMI, have shown potential. Furthermore, there is still much to explore in relation to drug carriers and regenerative medicine and treatment, with broad growth potential in the future. 


\section{Acknowledgements}

Not applicable.

\section{Funding}

This review was supported by the National Natural Science Foundation of China (grant no. 81800270).

\section{Availability of data and materials}

Not applicable.

\section{Authors' contributions}

JL contributed to the design and conception of the manuscript. $\mathrm{YZ}$ and $\mathrm{WZ}$ added contributions by revising and editing the final manuscript. All authors have read and approved the final version of the manuscript. Data authentication is not applicable.

\section{Ethics approval and consent to participate}

Not applicable.

\section{Patient consent for publication}

Not applicable.

\section{Competing interests}

The authors declare that they have no competing interests.

\section{References}

1. Mehta LS, Beckie TM, DeVon HA, Grines CL, Krumholz HM, Johnson MN, Lindley KJ, Vaccarino V, Wang TY, Watson KE, et al; American Heart Association Cardiovascular Disease in Women and Special Populations Committee of the Council on Clinical Cardiology, Council on Epidemiology and Prevention, Council on Cardiovascular and Stroke Nursing, and Council on Quality of Care and Outcomes Research: Acute Myocardial Infarction in Women: A Scientific Statement From the American Heart Association. Circulation 133: 916-947, 2016.

2. Reed GW, Rossi JE and Cannon CP: Acute myocardial infarction. Lancet 389: 197-210, 2017.

3. Frangogiannis NG: Cardiac fibrosis: Cell biological mechanisms, molecular pathways and therapeutic opportunities. Mol Aspects Med 65: 70-99, 2019.

4. Mitsos S, Katsanos K, Koletsis E, Kagadis GC, Anastasiou N, Diamantopoulos A, Karnabatidis D and Dougenis D: Therapeutic angiogenesis for myocardial ischemia revisited: Basic biological concepts and focus on latest clinical trials. Angiogenesis 15: $1-22,2012$.

5. Lorier G, Touriño $\mathrm{C}$ and Kalil RA: Coronary angiogenesis as an endogenous response to myocardial ischemia in adults. Arq Bras Cardiol 97: e140-e148, 2011.

6. Vandekeere S, Dewerchin M and Carmeliet P: Angiogenesis Revisited: An Overlooked Role of Endothelial Cell Metabolism in Vessel Sprouting. Microcirculation 22: 509-517, 2015.

7. Weinstein N, Mendoza L, Gitler I and Klapp J: A network model to explore the effect of the micro-environment on endothelial cell behavior during angiogenesis. Front Physiol 8: 960, 2017.

8. Frangogiannis NG: The extracellular matrix in myocardial injury, repair, and remodeling. J Clin Invest 127: 1600-1612, 2017.

9. Frangogiannis NG: Pathophysiology of myocardial infarction. Compr Physiol 5: 1841-1875, 2015.

10. Ferraro B, Leoni G, Hinkel R, Ormanns S, Paulin N, OrtegaGomez A, Viola JR, de Jong R, Bongiovanni D, Bozoglu T, et al: Pro-angiogenic macrophage phenotype to promote myocardial repair. J Am Coll Cardiol 73: 2990-3002, 2019.
11. Wang N, Liu C, Wang X, He T, Li L, Liang X, Wang L, Song L, Wei Y, Wu Q, et al: Hyaluronic acid oligosaccharides improve myocardial function reconstruction and angiogenesis against myocardial infarction by regulation of macrophages. Theranostics 9: 1980-1992, 2019.

12. Saraswati S, Marrow SMW, Watch LA and Young PP: Identification of a pro-angiogenic functional role for FSP1-positive fibroblast subtype in wound healing. Nat Commun 10: 3027, 2019.

13. Mouton AJ, Ma Y, Rivera Gonzalez OJ, Daseke MJ II, Flynn ER, Freeman TC, Garrett MR, DeLeon-Pennell KY and Lindsey ML: Fibroblast polarization over the myocardial infarction time continuum shifts roles from inflammation to angiogenesis. Basic Res Cardiol 114: 6, 2019.

14. Befani $\mathrm{C}$ and Liakos P: Hypoxia upregulates integrin gene expression in microvascular endothelial cells and promotes their migration and capillary-like tube formation. Cell Biol Int 41: 769-778, 2017.

15. Bartoszewski R, Moszyńska A, Serocki M, Cabaj A, Polten A, Ochocka R, Dell'Italia L, Bartoszewska S, Króliczewski J, Dąbrowski M, et al: Primary endothelial cell-specific regulation of hypoxia-inducible factor (HIF)-1 and HIF-2 and their target gene expression profiles during hypoxia. FASEB J 33: 7929-7941, 2019.

16. Zhang B, Niu W, Dong HY, Liu ML, Luo Y and Li ZC: Hypoxia induces endothelial mesenchymal transition in pulmonary vascular remodeling. Int J Mol Med 42: 270-278, 2018.

17. Ribatti D, Tamma R and Vacca A: Mast cells and angiogenesis in human plasma cell malignancies. Int J Mol Sci 20: 20, 2019.

18. Fetz AE, Radic MZ and Bowlin GL: Neutrophils in biomaterial-guided tissue regeneration: Matrix reprogramming for angiogenesis. Tissue Eng Part B Rev 27: 95-106, 2021.

19. Aldabbous L, Abdul-Salam V, McKinnon T, Duluc L, Pepke-Zaba J, Southwood M, Ainscough AJ, Hadinnapola C, Wilkins MR, Toshner M, et al: Neutrophil extracellular traps promote angiogenesis: Evidence from vascular pathology in pulmonary hypertension. Arterioscler Thromb Vasc Biol 36: 2078-2087, 2016

20. Mukai K, Tsai M, Saito H and Galli SJ: Mast cells as sources of cytokines, chemokines, and growth factors. Immunol Rev 282: 121-150, 2018.

21. Nishida Y, Yamada Y, Kanemaru H, Ohazama A, Maeda T and Seo K: Vascularization via activation of VEGF-VEGFR signaling is essential for peripheral nerve regeneration. Biomed Res 39: 287-294, 2018.

22. Chen Y, Zhao B, Zhu Y, Zhao H and Ma C: HIF-1-VEGF-Notch mediates angiogenesis in temporomandibular joint osteoarthritis. Am J Transl Res 11: 2969-2982, 2019.

23. Pitulescu ME, Schmidt I, Giaimo BD, Antoine T, Berkenfeld F, Ferrante F, Park H, Ehling M, Biljes D, Rocha SF, et al: Dll4 and Notch signalling couples sprouting angiogenesis and artery formation. Nat Cell Biol 19: 915-927, 2017.

24. Kume T: Ligand-dependent Notch signaling in vascular formation. Adv Exp Med Biol 727: 210-222, 2012.

25. Gallo S, Sala V, Gatti S and Crepaldi T: Cellular and molecular mechanisms of HGF/Met in the cardiovascular system. Clin Sci (Lond) 129: 1173-1193, 2015.

26. Thavapalachandran S, Grieve SM, Hume RD, Le TY, Raguram K, Hudson JE, Pouliopoulos J, Figtree GA, Dye RP, Barry AM, et al: Platelet-derived growth factor-AB improves scar mechanics and vascularity after myocardial infarction. Sci Transl Med 12: 12 , 2020.

27. Liu S, Chen J, Shi J, Zhou W, Wang L, Fang W, Zhong Y, Chen X, Chen Y, Sabri A, et al: M1-like macrophage-derived exosomes suppress angiogenesis and exacerbate cardiac dysfunction in a myocardial infarction microenvironment. Basic Res Cardiol 115: 22,2020 .

28. Zhang Z, Coutinho AE, Man TY, Kipari TM, Hadoke PW, Salter DM, Seckl JR and Chapman KE: Macrophage 11ß-HSD-1 deficiency promotes inflammatory angiogenesis. J Endocrinol 234: 291-299, 2017.

29. Hueso L, Rios-Navarro C, Ruiz-Sauri A, Chorro FJ, Nunez J, Sanz MJ, Bodi V and Piqueras L: Dynamics and implications of circulating anti-angiogenic VEGF-A165b isoform in patients with ST-elevation myocardial infarction. Sci Rep 7: 9962, 2017.

30. Rychli K, Kaun C, Hohensinner PJ, Dorfner AJ, Pfaffenberger S, Niessner A, Bauer M, Dietl W, Podesser BK, Maurer G, et al: The anti-angiogenic factor PEDF is present in the human heart and is regulated by anoxia in cardiac myocytes and fibroblasts. J Cell Mol Med 14: 198-205, 2010. 
31. Sakamoto S, Matsuura K, Masuda S, Hagiwara N and Shimizu T: Heart-derived fibroblasts express LYPD-1 and negatively regulate angiogenesis in rat. Regen Ther 15: 27-33, 2020

32. Jiang L, Jia M, Wei X, Guo J, Hao S, Mei A, Zhi X, Wang X, Li Q, Jin J, et al: Bach1-induced suppression of angiogenesis is dependent on the BTB domain. EBioMedicine 51: 102617, 2020.

33. Xie Y, Sheng W, Xiang J, Ye Z, Zhu Y, Chen X and Yang J: Recombinant human IL-24 suppresses lung carcinoma cell growth via induction of cell apoptosis and inhibition of tumor angiogenesis. Cancer Biother Radiopharm 23: 310-320, 2008.

34. Wang Z, Lv J and Zhang T: Combination of IL-24 and cisplatin inhibits angiogenesis and lymphangiogenesis of cervical cancer xenografts in a nude mouse model by inhibiting VEGF, VEGF-C and PDGF-B. Oncol Rep 33: 2468-2476, 2015.

35. Nisari M, Ulger H, Unur E, Karaca O and Ertekin T: Effect of interleukin 12 (IL-12) on embryonic development and yolk sac vascularisation. Bratisl Lek Listy 115: 532-537, 2014.

36. Ding DC, Shyu WC and Lin SZ: Mesenchymal stem cells. Cell Transplant 20: 5-14, 2011.

37. Uccelli A, Moretta L and Pistoia V: Mesenchymal stem cells in health and disease. Nat Rev Immunol 8: 726-736, 2008.

38. Konoplyannikov M, Kotova S, Baklaushev V, Konoplyannikov A, Kalsin V, Timashev P and Troitskiy A: Mesenchymal stem cell therapy for ischemic heart disease: Advances and challenges. Curr Pharm Des 24: 3132-3142, 2018.

39. Mathew SA, Naik C, Cahill PA and Bhonde RR: Placental mesenchymal stromal cells as an alternative tool for therapeutic angiogenesis. Cell Mol Life Sci 77: 253-265, 2020.

40. Kachgal S and Putnam AJ: Mesenchymal stem cells from adipose and bone marrow promote angiogenesis via distinct cytokine and protease expression mechanisms. Angiogenesis 14: 47-59, 2011.

41. Assis-Ribas T, Forni MF, Winnischofer SM, Sogayar MC and Trombetta-Lima M: Extracellular matrix dynamics during mesenchymal stem cells differentiation. Dev Biol 437: 63-74, 2018.

42. Huang W, Wang T, Zhang D, Zhao T, Dai B, Ashraf A, Wang X, Xu M, Millard RW, Fan GC, et al: Mesenchymal stem cells overexpressing CX7CR4 attenuate remodeling of postmyocardial infarction by releasing matrix metalloproteinase-9. Stem Cells Dev 21: 778-789, 2012.

43. Gnecchi M, Danieli P, Malpasso G and Ciuffreda MC: Paracrine mechanisms of mesenchymal stem cells in tissue repair. Methods Mol Biol 1416: 123-146, 2016.

44. Gunawardena TNA, Rahman MT, Abdullah BJJ and Abu Kasim NH: Conditioned media derived from mesenchymal stem cell cultures: The next generation for regenerative medicine. J Tissue Eng Regen Med 13: 569-586, 2019.

45. Wang Z, Zheng L, Lian C, Qi Y, Li W and Wang S: Human umbilical cord-derived mesenchymal stem cells relieve hind limb ischemia by promoting angiogenesis in mice. Stem Cells Dev 28: 1384-1397, 2019.

46. Ryu S, Lee SH, Kim SU and Yoon BW: Human neural stem cells promote proliferation of endogenous neural stem cells and enhance angiogenesis in ischemic rat brain. Neural Regen Res 11: 298-304, 2016.

47. Davidson SM and Yellon DM: Exosomes and cardioprotection A critical analysis. Mol Aspects Med 60: 104-114, 2018.

48. Zhang Y, Bi J, Huang J, Tang Y, Du S and Li P: Exosome: A review of its classification, isolation techniques, storage, diagnostic and targeted Therapy applications. Int J Nanomedicine 15 : 6917-6934, 2020 .

49. Koritzinsky EH, Street JM, Star RA and Yuen PS: Quantification of exosomes. J Cell Physiol 232: 1587-1590, 2017.

50. Witwer KW, Soekmadji C, Hill AF, Wauben MH, Buzás EI, Di Vizio D, Falcon-Perez JM, Gardiner C, Hochberg F, Kurochkin IV, et al: Updating the MISEV minimal requirements for extracellular vesicle studies: Building bridges to reproducibility. J Extracell Vesicles 6: 1396823, 2017.

51. Kawamoto A and Losordo DW: Endothelial progenitor cells for cardiovascular regeneration. Trends Cardiovasc Med 18: 33-37, 2008.

52. Zeng CY, Xu J, Liu X and Lu YQ: Cardioprotective roles of endothelial progenitor cell-derived exosomes. Front Cardiovasc Med 8: 717536, 2021

53. Pan MC, Lin XY, Wang H, Chen YF and Leng M: Research advances on the roles of exosomes derived from vascular endothelial progenitor cells in wound repair. Zhonghua Shao Shang Za Zhi Zhonghua Shao Shang Za Zhi 36: 883-886, 2020 (In Chinese)
54. Xing Z, Zhao C, Liu H and Fan Y: Endothelial progenitor cell-derived extracellular vesicles: A novel candidate for regenerative medicine and disease treatment. Adv Healthc Mater 9: e2000255, 2020.

55. Ke X, Yang D, Liang J, Wang X, Wu S, Wang X and Hu C: Human endothelial progenitor cell-derived exosomes increase proliferation and angiogenesis in cardiac fibroblasts by promoting the mesenchymal-endothelial transition and reducing high mobility group box 1 protein B1 expression. DNA Cell Biol 36: 1018-1028, 2017.

56. Wang J, Liu H, Chen S, Zhang W, Chen Y and Yang Y: Moderate exercise has beneficial effects on mouse ischemic stroke by enhancing the functions of circulating endothelial progenitor cell-derived exosomes. Exp Neurol 330: 113325, 2020.

57. Wang Y, Zhao R, Shen C, Liu W, Yuan J, Li C, Deng W, Wang Z, Zhang W, Ge J, et al: Exosomal CircHIPK3 released from hypoxia-induced cardiomyocytes regulates cardiac angiogenesis after myocardial infarction. Oxid Med Cell Longev 2020: 8418407,2020

58. Wang Y, Zhao R, Liu W, Wang Z, Rong J, Long X, Liu Z, Ge J and Shi B: Exosomal circHIPK3 released from hypoxia-pretreated cardiomyocytes regulates oxidative damage in cardiac microvascular endothelial cells via the miR-29a/IGF-1 pathway. Oxid Med Cell Longev 2019: 7954657, 2019.

59. Yan B, Zhang Y, Liang C, Liu B, Ding F, Wang Y, Zhu B, Zhao R, Yu XY and Li Y: Stem cell-derived exosomes prevent pyroptosis and repair ischemic muscle injury through a novel exosome/ circHIPK3/ FOXO3a pathway. Theranostics 10: 6728-6742, 2020.

60. Li H, Liao Y, Gao L, Zhuang T, Huang Z, Zhu H and Ge J: Coronary serum exosomes derived from patients with myocardial ischemia regulate angiogenesis through the miR-939-mediated nitric oxide signaling pathway. Theranostics 8: 2079-2093, 2018.

61. Xu J, Bai S, Cao Y, Liu L, Fang Y, Du J, Luo L, Chen M, Shen B and Zhang Q: miRNA-221-3p in endothelial progenitor cell-derived exosomes accelerates skin wound healing in diabetic mice. Diabetes Metab Syndr Obes 13: 1259-1270, 2020.

62. Chen K, Yu T and Wang X: Inhibition of circulating exosomal miRNA-20b-5p accelerates diabetic wound repair. Int J Nanomedicine 16: 371-381, 2021

63. Ren S, Chen J, Duscher D, Liu Y, Guo G, Kang Y, Xiong H, Zhan P, Wang Y, Wang C, et al: Microvesicles from human adipose stem cells promote wound healing by optimizing cellular functions via AKT and ERK signaling pathways. Stem Cell Res Ther 10: 47, 2019.

64. Xiong Y, Chen L, Yan C, Zhou W, Endo Y, Liu J, Hu L, Hu Y, Mi B and Liu G: Circulating Exosomal miR-20b-5p inhibition restores Wnt9b signaling and reverses diabetes-associated impaired wound healing. Small 16: e1904044, 2020.

65. Ni J, Liu X, Yin Y, Zhang P, Xu YW and Liu Z: Exosomes derived from TIMP2-modified human umbilical cord mesenchymal stem cells enhance the repair effect in rat model with myocardial infarction possibly by the Akt/Sfrp2 pathway. Oxid Med Cell Longev 2019: 1958941, 2019

66. Sun J, Shen H, Shao L, Teng X, Chen Y, Liu X, Yang Z and Shen Z: HIF-1 $\alpha$ overexpression in mesenchymal stem cell-derived exosomes mediates cardioprotection in myocardial infarction by enhanced angiogenesis. Stem Cell Res Ther 11: 373, 2020.

67. Gong XH, Liu H, Wang SJ, Liang SW and Wang GG: Exosomes derived from SDF1-overexpressing mesenchymal stem cells inhibit ischemic myocardial cell apoptosis and promote cardiac endothelial microvascular regeneration in mice with myocardial infarction. J Cell Physiol 234: 13878-13893, 2019.

68. Wang X, Chen Y, Zhao Z, Meng Q, Yu Y, Sun J, Yang Z, Chen Y, Li J, Ma T, et al: Engineered exosomes with ischemic myocardium-targeting peptide for targeted therapy in myocardial infarction. J Am Heart Assoc 7: e008737, 2018.

69. Youn SW, Li Y, Kim YM, Sudhahar V, Abdelsaid K, Kim HW, Liu Y, Fulton DJ, Ashraf M, Tang Y, et al: Modification of cardiac progenitor cell-derived exosomes by miR-322 provides protection against myocardial infarction through Nox2-dependent angiogenesis. Antioxidants (Basel) 8: 18, 2019.

70. Pan J, Alimujiang M, Chen Q, Shi $\mathrm{H}$ and Luo X: Exosomes derived from miR-146a-modified adipose-derived stem cells attenuate acute myocardial infarction-induced myocardial damage via downregulation of early growth response factor 1 . J Cell Biochem 120: 4433-4443, 2019.

71. Fan C, Joshi J, Li F, Xu B, Khan M, Yang J and Zhu W: Nanoparticle-mediated drug delivery for treatment of ischemic heart disease. Front Bioeng Biotechnol 8: 687, 2020 
72. Zhang N, Song Y, Huang Z, Chen J, Tan H, Yang H, Fan M, Li Q, Wang Q, Gao J, et al: Monocyte mimics improve mesenchymal stem cell-derived extracellular vesicle homing in a mouse MI/RI model. Biomaterials 255: 120168, 2020.

73. Ho YT, Poinard B and Kah JC: Nanoparticle drug delivery systems and their use in cardiac tissue therapy. Nanomedicine (Lond) 11: 693-714, 2016.

74. Li Z, Zhou X, Wei M, Gao X, Zhao L, Shi R, Sun W, Duan Y, Yang $G$ and Yuan L: In vitro and in vivo RNA inhibition by CD9-HuR functionalized exosomes encapsulated with miRNA or CRISPR/dCas9. Nano Lett 19: 19-28, 2019.

75. Kojima R, Bojar D, Rizzi G, Hamri GC, El-Baba MD, Saxena P, Ausländer S, Tan KR and Fussenegger M: Designer exosomes produced by implanted cells intracerebrally deliver therapeutic cargo for Parkinson's disease treatment. Nat Commun 9: 1305 , 2018.

76. Xiang Gu G, Su I, Sharma S, Voros JL, Qin Z and Buehler MJ: Three-dimensional-printing of bio-inspired composites. J Biomech Eng 138: 021006, 2016.

77. Chattopadhyay S and Raines RT: Review collagen-based biomaterials for wound healing. Biopolymers 101: 821-833, 2014.

78. Smagul S, Kim Y, Smagulova A, Raziyeva K, Nurkesh A and Saparov A: Biomaterials loaded with growth factors/cytokines and stem cells for cardiac tissue regeneration. Int J Mol Sci 21: $21,2020$.

79. Oduk Y, Zhu W, Kannappan R, Zhao M, Borovjagin AV, Oparil S and Zhang JJ: VEGF nanoparticles repair the heart after myocardial infarction. Am J Physiol Heart Circ Physiol 314: $\mathrm{H} 278-\mathrm{H} 284,2018$

80. Liu Y, Li P, Qiao C, Wu T, Sun X, Wen M and Zhang W: Chitosan hydrogel enhances the therapeutic efficacy of bone marrow-derived mesenchymal stem cells for myocardial infarction by alleviating vascular endothelial cell pyroptosis. J Cardiovasc Pharmacol 75: 75-83, 2020.

81. Yuan Z, Tsou YH, Zhang XQ, Huang S, Yang Y, Gao M, Ho W Zhao Q, Ye X and Xu X: Injectable citrate-based hydrogel as an angiogenic biomaterial improves cardiac repair after myocardial infarction. ACS Appl Mater Interfaces 11: 38429-38439, 2019.

82. Song C, Zhang X, Wang L, Wen F, Xu K, Xiong W, Li C, Li B, Wang Q, Xing MM, et al: An injectable conductive three-dimensional elastic network by tangled surgical-suture spring for heart repair. ACS Nano 13: 14122-14137, 2019.

83. Chachques JC, Lila N, Soler-Botija C, Martinez-Ramos C, Valles A, Autret G, Perier MC, Mirochnik N, Monleon-Pradas M, Bayes-Genis A, et al: Elastomeric cardiopatch scaffold for myocardial repair and ventricular support. Eur J Cardiothorac Surg 57: 545-555, 2020

84. Wang X, Wang L, Wu Q, Bao F, Yang H, Qiu X and Chang J: Chitosan/calcium silicate cardiac patch stimulates cardiomyocyte activity and myocardial performance after infarction by synergistic effect of bioactive ions and aligned nanostructure. ACS Appl Mater Interfaces 11: 1449-1468, 2019.

85. Sondermeijer HP, Witkowski P, Seki T, van der Laarse A, Itescu S and Hardy MA: RGDfK-peptide modified alginate scaffold for cell transplantation and cardiac neovascularization. Tissue Eng Part A 24: 740-751, 2018

86. Nasser M, Wu Y, Danaoui Y and Ghosh G: Engineering microenvironments towards harnessing pro-angiogenic potential of mesenchymal stem cells. Mater Sci Eng C 102: 75-84, 2019.

87. Dastagir K, Dastagir N, Limbourg A, Reimers K, Strauss S and Vogt PM: In vitro construction of artificial blood vessels using spider silk as a supporting matrix. J Mech Behav Biomed Mater 101: 103436, 2020.

88. Guo HF, Dai WW, Qian DH, Qin ZX, Lei Y, Hou XY and Wen C: A simply prepared small-diameter artificial blood vessel that promotes in situ endothelialization. Acta Biomater 54: 107-116, 2017.

89. Yifa O, Weisinger K, Bassat E, Li H, Kain D, Barr H, Kozer N, Genzelinakh A, Rajchman D, Eigler T, et al: The small molecule Chicago Sky Blue promotes heart repair following myocardial infarction in mice. JCI Insight 4: 4, 2019.

90. Huang FY, Xia TL, Li JL, Li CM, Zhao ZG, Lei WH, Chen L, Liao YB, Xiao D, Peng Y, et al: The bifunctional SDF-1-AnxA5 fusion protein protects cardiac function after myocardial infarction. J Cell Mol Med 23: 7673-7684, 2019.

91. Yuan Z, Kang L, Wang Z, Chen A,Zhao Q and Li H: $17 \beta$-estradiol promotes recovery after myocardial infarction by enhancing homing and angiogenic capacity of bone marrow-derived endothelial progenitor cells through ER $\alpha$-SDF-1/CXCR4 crosstalking. Acta Biochim Biophys Sin (Shanghai) 50: 1247-1256, 2018.
92. Popa MA, Mihai MC, Constantin A, Suică V, Tucureanu C, Costache R, Antohe F, Dubey RK and Simionescu M Dihydrotestosterone induces pro-angiogenic factors and assists homing of MSC into the cardiac tissue. J Mol Endocrinol 60: 1-15, 2018.

93. Liao Q, Qu S, Tang LX, Li LP, He DF, Zeng CY and Wang WE: Irisin exerts a therapeutic effect against myocardial infarction via promoting angiogenesis. Acta Pharmacol Sin 40: 1314-1321, 2019.

94.Lindsey ML, Iyer RP, Zamilpa R, Yabluchanskiy A DeLeon-Pennell KY, Hall ME, Kaplan A, Zouein FA, Bratton D, Flynn ER, et al: A novel collagen matricryptin reduces left ventricular dilation post-myocardial infarction by promoting scar formation and angiogenesis. J Am Coll Cardiol 66: 1364-1374, 2015.

95. Korf-Klingebiel M, Reboll MR, Grote K, Schleiner H, Wang Y, Wu X, Klede S, Mikhed Y, Bauersachs J, Klintschar M, et al: Heparan sulfate-editing extracellular sulfatases enhance vegf bioavailability for ischemic heart repair. Circ Res 125: 787-801, 2019.

96. Garikipati VNS, Verma SK, Cheng Z, Liang D, Truongcao MM, Cimini M, Yue Y, Huang G, Wang C, Benedict C, et al: Circular RNA CircFndc3b modulates cardiac repair after myocardial infarction via FUS/VEGF-A axis. Nat Commun 10: 4317, 2019.

97. Ju X, Xue D, Wang T, Ge B, Zhang Y and Li Z: Catalpol promotes the survival and VEGF secretion of bone marrow-derived stem cells and their role in myocardial repair after myocardial infarction in rats. Cardiovasc Toxicol 18: 471-481, 2018.

98. Zhai S, Zhang XF, Lu F, Chen WG, He X, Zhang CF, Wang CZ and Yuan CS: Chinese medicine GeGen-DanShen extract protects from myocardial ischemic injury through promoting angiogenesis via up-regulation of VEGF/VEGFR2 signaling pathway. J Ethnopharmacol 267: 113475, 2021.

99. Li Y, Zhang Y, Wen M, Zhang J, Zhao X, Zhao Y and Deng J: Ginkgo biloba extract prevents acute myocardial infarction and suppresses the inflammation and apoptosis regulating p38 mitogen activated protein kinases, nuclear factor- $\mathrm{KB}$ and $\mathrm{B}$ cell lymphoma 2 signaling pathways. Mol Med Rep 16: 3657-3663, 2017.

100. Ho L, van Dijk M, Chye STJ, Messerschmidt DM, Chng SC, Ong S, Yi LK, Boussata S, Goh GH, Afink GB, et al: ELABELA deficiency promotes preeclampsia and cardiovascular malformations in mice. Science 357: 707-713, 2017.

101. Jin L, Pan Y, Li Q, Li J and Wang Z: Elabela gene therapy promotes angiogenesis after myocardial infarction. J Cell Mol Med 25: 8537-8545, 2021.

102. Chen HK, Hung HF, Shyu KG, Wang BW, Sheu JR, Liang YJ, Chang CC and Kuan P: Combined cord blood stem cells and gene therapy enhances angiogenesis and improves cardiac performance in mouse after acute myocardial infarction. Eur J Clin Invest 35: 677-686, 2005.

103. Czymai T, Viemann D, Sticht C, Molema G, Goebeler M and Schmidt M: FOXO3 modulates endothelial gene expression and function by classical and alternative mechanisms. J Biol Chem 285: 10163-10178, 2010.

104. Yan P, Li Q, Wang L, Lu P, Suzuki K, Liu Z, Lei J, Li W, He X, Wang S, et al: FOXO3-engineered human ESC-derived vascular cells promote vascular protection and regeneration. Cell Stem Cell 24: 447-461.e8, 2019.

105. Wu D, Liu Y, Liu X, Liu W, Shi H, Zhang Y, Zou L and Zhao Y: Heme oxygenase-1 gene modified human placental mesenchymal stem cells promote placental angiogenesis and spiral artery remodeling by improving the balance of angiogenic factors in vitro. Placenta 99: 70-77, 2020.

106. Shevchenko EK, Makarevich PI, Tsokolaeva ZI, Boldyreva MA, Sysoeva VY, Tkachuk VA and Parfyonova YV: Transplantation of modified human adipose derived stromal cells expressing VEGF165 results in more efficient angiogenic response in ischemic skeletal muscle. J Transl Med 11: 138, 2013.

107. Mushimiyimana I, Tomas Bosch V, Niskanen H, Downes NL, Moreau PR, Hartigan K, Ylä-Herttuala S, Laham-Karam N and Kaikkonen MU: Genomic landscapes of noncoding RNAs regulating VEGFA and VEGFC expression in endothelial cells. Mol Cell Biol 41: e0059420, 2021.

108. Zhen S, Qiang R, Lu J, Tuo X, Yang X and Li X: TGF- $\beta 1$-based CRISPR/Cas9 gene therapy attenuate radiation-induced lung injury. Curr Gene Ther: Dec 29, 2020 (Epub ahead of print). doi: 10.2174/1566523220666201230100523.

109. van der Laan AM, Piek JJ and van Royen N: Targeting angiogenesis to restore the microcirculation after reperfused MI. Nat Rev Cardiol 6: 515-523, 2009. 
110. Tarantini G, Ramondo A, Napodano M, Favaretto E, Gardin A, Bilato C, Nesseris G, Tarzia V, Cademartiri F, Gerosa G, et al PCI versus CABG for multivessel coronary disease in diabetics. Catheter Cardiovasc Interv 73: 50-58, 2009.

111. Montrief T, Koyfman A and Long B: Coronary artery bypass graft surgery complications: A review for emergency clinicians. Am J Emerg Med 36: 2289-2297, 2018.

112. Wang L, Huang S, Li S, Li M, Shi J, Bai W, Wang Q, Zheng L and Liu Y: Efficacy and safety of umbilical cord mesenchymal stem cell therapy for rheumatoid arthritis patients: A prospective phase I/II study. Drug Des Devel Ther 13: 4331-4340, 2019.

113. Watanabe Y, Tsuchiya A and Terai S: The development of mesenchymal stem cell therapy in the present, and the perspective of cell-free therapy in the future. Clin Mol Hepatol 27: 70-80, 2021.

114. Yu B, Zhang X and Li X: Exosomes derived from mesenchymal stem cells. Int J Mol Sci 15: 4142-4157, 2014.
115. Yamashita T, Takahashi Y and Takakura Y: Possibility of exosome-based therapeutics and challenges in production of exosomes eligible for therapeutic application. Biol Pharm Bull 41: 835-842, 2018

116. He X, Wang Q, Zhao Y, Zhang H, Wang B, Pan J, Li J, Yu H, Wang L, Dai J, et al: Effect of intramyocardial grafting collagen scaffold with mesenchymal stromal cells in patients with chronic ischemic heart disease: A randomized clinical trial. JAMA Netw Open 3: e2016236, 2020.

117. Topaloğlu Demir F, Özkök Akbulut T, Kıvanç Altunay İ, Aytekin S, Oğuz Topal İ, Kara Polat A, Özkur E and Karadağ AS: Evaluation of the adverse effects of biological agents used in the treatment of psoriasis: A multicenter retrospective cohort study. Dermatol Ther 33: e14216, 2020. 